\title{
Germ cell migration under GAGA-factor control
}

\author{
A. Khruscheva ${ }^{1,2}$, K. Ahmetova ${ }^{1}$, S. Fedorova ${ }^{1,2}$, E. Baricheva $^{1}$, N. Dorogova ${ }^{1 *}$ \\ ${ }^{1}$ Institute of Cytology and Genetics SB RAS, Novosibirsk, Russia \\ ${ }^{2}$ Novosibirsk National Research State University, Novosibirsk, Russia \\ *e-mail:dorogova@bionet.nsc.ru
}

Key words: germ cells, cell migration, GAGA-factor, $\operatorname{Trl}$ gene, Drosophila

Motivation and Aim: Germ cells migration is an important stage of gonadogenesis and normal development of precursors of germ cells. This process occurs in early embryogenesis and it is carried out by the ability of the germ cells (GC) to move individually and actively, using specialized structures and penetrating the surrounding cellular barrier (epithelium). Molecular-genetic nature of GCs migration is evolutionarily conservative and has high homology in different cell types, organs and tissues, and it is similar in many aspects with the movement of the immune system cells and metastatic tumors. This circumstance explains the interest in this research topic and confirms its relevance.

Methods and Algorithms: We used the genetic model of Drosophila melanogaster, cytological methods and bioinformatic analysis.

Results: In this study, we performed a detailed cytological analysis of the early stages of germ cell development in the mutant background of the Trl gene that encodes GAGAfactor. We showed that the mutation causes premature morphological transformation of the early germ cells into actively migrating cells and, consequently, promote their ectopic migration inside the embryo. The first signs of active migration in mutants observed already at the stage of the cellular blastoderm. Part of the germ cells becomed amoeboid and migrated through a single-layered epithelium. These cells migrated chaotically, disoriented and did not reach the gonadal region. Premature migration leads to a reduction in the germ cells number in the adult flies. The character of Trl gene expression indicates its activity in epithelial blastoderm cells, some of which contact with primordial germ cells. It is the most likely that anomalies in the migration are associated with a mutant effect in surrounding somatic epithelial cells. Since the GAGA-factor regulated the expression of diversity genes, its effect mediated through the activation of target genes. We analyzed both expression patterns and regulatory regions of genes that expressed during embryonic GC migration and revealed 25 potential GAGA-target genes.

Conclusion: Thus, the GAGA-factor influences the migration of the embryonic GCs through the regulation of target gene expression in their somatic environment. The mutant effect in somatic cells led to an early activation of the migration program and the premature trans-epithelial GCs migration. The phenomenon of premature migration demonstrates that the somatic environment, in particular the epithelial cells, do not simply form a substrate for GCs migration, but can also influence this process in a regulatory manner.

Acknowledgements: Supported by the RFBR 18-34-00321mol_a (A. Khruscheva, K. Ahmetova) and the State Project No. 0324-2018-0019 (S. Fedorova, E. Baricheva, N. Dorogova). 